\title{
INFERENCE ON NONPARAMETRICALLY TRENDING TIME SERIES WITH FRACTIONAL ERRORS
}

\author{
P. M. Robinson* \\ London School of Economics
}

Discussion paper No. EM/2009/532 October 2008
The Suntory Centre

Suntory and Toyota International Centres for Economics and Related Disciplines London School of Economics and Political Science Houghton Street London WC2A 2AE Tel: 02079556679

\footnotetext{
* Submission intended for the Paul Newbold Special Issue. E-mail address: p.m.robinson@Ise.ac.uk. This research was supported by ESRC Grant RES-062-23-0036. I am grateful for the comments of three referees and Rob Taylor.
} 


\begin{abstract}
The central limit theorem for nonparametric kernel estimates of a smooth trend, with linearly-generated errors, indicates asymptotic independence and homoscedasticity across fixed points, irrespective of whether disturbances have short memory, long memory, or antipersistence. However, the asymptotic variance depends on the kernel function in a way that varies across these three circumstances, and in the latter two involves a double integral that cannot necessarily be evaluated in closed form. For a particular class of kernels, we obtain analytic formulae. We discuss extensions to more general settings, including ones involving possible cross-sectional or spatial dependence.
\end{abstract}

Proposed running head:

Nonparametrically Trending Time Series

Professor Peter M Robinson

Department of Economics

London School of Economics

Houghton Street

London WC2A 2AE

UK

phone: +44-(0)20-7955-7516

fax: +44-(0)20-7955-6592

(C) The author. All rights reserved. Short sections of text, not to exceed two paragraphs, may be quoted without explicit permission provided that full credit, including (c) notice, is given to the source. 


\section{Introduction}

Deterministic trend estimation for time series and panel data can be carried out in numerous ways. With macroeconomic time series, use of a polynomial-in-time trend, even a linear trend, is still very common. Classically, polynomial order can be very simply and exactly (in finite samples) tested when the model is expressed in terms of orthogonal polynomials, and disturbances are independent, identically distributed and Gaussian, while on the other hand, least squares estimates of the coefficients are asymptotically efficient (in the Gauss-Markov sense) even in the presence of general short-range dependence in possibly non-Gaussian stationary disturbances, the technical requirement being that the disturbance spectral density is continuous and positive at zero frequency. Polynomial time trends are also frequently associated with investigation of unit root behaviour of the stochastic component. They are also convenient in panel data, especially when the time dimension is not long.

Use of a polynomial, or any parametric function, is inevitably subject to the consequences of misspecification: typically, large-sample rules of statistical inference are rendered invalid. Moreover, viewed as approximations to general functions of unbounded support, polynomials do not have very good properties. Given a long time series, an attractive alternative to a high-degree polynomial, and one that is no less ad hoc, is kernel smoothing. Here, the trend is viewed as a smooth, nonparametric function of time.

When the focus is on estimation of the trend, rather than its removal, there is also interest in attaching a measure of variation. If one is prepared to consider estimates at a number of time points that is small, in the sense of remaining fixed as series length $T$ increases, we need only approximate a finite-dimensional joint distribution. Moreover, the normal approximation, as $T \rightarrow \infty$, to the limit distribution can be not only multivariate normal but spherical, in other words, having covariance matrix proportional to the identity. Thus interval estimation promises to be computationally convenient. Moreover, these kind of properties can hold not only when disturbances are independent or short range dependent, but even when they have stationary long memory or antipersistence.

To fix ideas, consider the scalar observable sequence $y_{t}, t=1,2, \ldots, T$, described by

$$
y_{t}=r\left(\frac{t}{T}\right)+x_{t}, t=1, \ldots, T,
$$

where $r$ is a smooth function on the unit interval $(0,1]$, and $x_{t}$ is a zero-mean covariance stationary process. Of course $y_{t}$ also depends on $T$, and so the $y_{t}, t=1, \ldots, T$, form a triangular array as $T$ increases, but as is common our notation ignores this. The dependence of the trend $r(t / T)$ on $T$ is required in order to produce a useful asymptotic theory for locally-smoothed estimates of $r$.

For a fixed point $\tau$, consider the kernel estimate

$$
\hat{r}(\tau)=\frac{1}{T q} \sum_{t=1}^{T} k\left(\frac{T \tau-t}{T q}\right) y_{t},
$$

for some $\tau \in(0,1)$, where $q=q_{T}$ is a positive, user-chosen, bandwidth sequence, 
and $k$ is a kernel function satisfying at least

$$
\int_{-\infty}^{\infty} k(u) d u=1
$$

We consider $\hat{r}\left(\tau_{s}\right), s=1, \ldots, S$, where the $\tau_{s}$ are distinct values in $(0,1)$ that remain fixed as $T \rightarrow \infty$, as does $S$.

The $\hat{r}\left(\tau_{s}\right)$, after centring at $r\left(\tau_{s}\right)$ and suitable normalization, can be shown to be asymptotically independently and normally distributed under regularity conditions that, so far as second-order structure of $x_{t}$ is concerned, involve only the local-to-zero character of its spectral density, $f(\lambda)$. We assume, following Assumption 1 of Robinson (1997), that it has form

$$
f(\lambda)=g(\lambda) h(\lambda), \quad-\pi<\lambda \leq \pi,
$$

where $g$ is an even, nonnegative function that is continuous at $\lambda=0$, and $h$ is an integrable function such that

$$
\delta_{j}=\int_{-\pi}^{\pi} h(\lambda) \cos (j \lambda) d \lambda
$$

satisfies, for some $d \in\left(-\frac{1}{2}, \frac{1}{2}\right)$,

$$
\begin{aligned}
\delta_{j} & \sim \phi(d) j^{2 d-1}, \text { as } j \rightarrow \infty, \text { if } 0<|d|<\frac{1}{2}, \\
& =2 \pi \Delta_{j 0}, \text { if } d=0,
\end{aligned}
$$

where $\Delta_{j 0}$ is the Kronecker delta (so $\Delta_{00}=1, \Delta_{j 0}=0$ for $j \neq 0$ ), and

$$
\begin{aligned}
& \phi(d)=2 \Gamma(1-2 d) \sin (\pi d), \\
& h(0)=0, \text { if } d \in\left(-\frac{1}{2}, 0\right) .
\end{aligned}
$$

When $d=0$ we deduce that $h(\lambda)=1,-\pi<\lambda \leq \pi$, and thus $f(\lambda)=g(\lambda)$, so that $f(\lambda)$ satisfies a mild form of short memory condition. More generally, we can relate the assumptions to the property

$$
h(\lambda) \sim \lambda^{-2 d}, \text { as } \lambda \rightarrow 0+,
$$

which implies

$$
f(\lambda) \sim G \lambda^{-2 d}, \text { as } \lambda \rightarrow 0+,
$$

denoting

$$
G=g(0) .
$$

When $d \in\left(0, \frac{1}{2}\right),(1.10)$ is equivalent to (1.6) if the $\delta_{j}$ are quasi-monotonically convergent to zero (Yong, 1974, Theorem III-16). Here, $f(\lambda)$ diverges at $\lambda=0$, and $x_{t}$ is said to have long memory. For $d \in\left(-\frac{1}{2}, 0\right),(1.8)$ and(1.9) together imply (1.10) (Yong, 1974, Theorem III-31). Here, $f(0)=0$ and $x_{t}$ is said to have negative memory, or antipersistence.

These prescriptions do not entail any parametric assumption on the secondorder properties of $x_{t}$, in similar spirit to our nonparametric treatment of trend. Moreover, not only are we likely to be ignorant of the precise value of $d$, but in case it is non-zero we may not even know whether it is positive or negative. 
Thus there is advantage in an asymptotic distribution theory which assumes only that $d$ lies in the stationary and invertible region $\left(-\frac{1}{2}, \frac{1}{2}\right)$.

Robinson (1997) developed such a theory, under the following additional conditions. First $r(u)$ satisfies a Lipschitz condition of degree $\gamma, 0<\gamma \leq 1$, or $r(u)$ is differentiable with derivative satisfying a Lipschitz condition of degree $\gamma-1,1<\gamma \leq 2$. Next, $x_{t}=\Sigma_{j=-\infty}^{\infty} c_{j} \varepsilon_{t-j}, \Sigma_{j=-\infty}^{\infty} c_{j}^{2}<\infty$, for $t=0, \pm 1, \ldots$, where the $\varepsilon_{t}^{2}$ are uniformly integrable and $E\left(\varepsilon_{t} \mid \mathcal{F}_{t-1}\right)=0, \mathrm{E}\left(\varepsilon_{t}^{2} \mid \mathcal{F}_{t-1}\right)=1$, a.s., $t=0, \pm 1, \ldots$, where $\mathcal{F}_{t}$ is the $\sigma$-field of events generated by $\left\{\varepsilon_{s}, s \leq t\right\}$. Next, $k(u)$ is even, eventually monotonically nonincreasing, almost everywhere differentiable, and in addition to (1.3), satisfies $k(u)=O\left(\left(1+u^{2}\right)^{-1}\right), k^{\prime}(u)=$ $O\left(\left(1+|u|^{1+\eta}\right)^{-1}\right)$ for some $\eta>0, k^{\prime}(u)$ denoting the derivative of $k(u)$ where it exists. Finally, $(T q)^{-1}+T^{\frac{1}{2}-d} q^{\frac{1}{2}-d+\gamma} \rightarrow 0$, as $T \rightarrow 0$.

The linear process assumption on $x_{t}$ might be replaced by a suitable mixing condition when $d=0$ (see e.g. Roussas, Tran and Ioannides (1992)), while for $d \in\left(0, \frac{1}{2}\right) x_{t}$ might instead be represented as a nonlinear function of a Gaussian long memory process (cf. Fox and Taqqu, 1985). However, the linearity assumption conveniently covers simultaneously short memory, long memory, and antipersistence.

Under the above conditions, Theorem 1 of Robinson (1997) states that the

$$
(T q)^{\frac{1}{2}-d}\left\{\hat{r}\left(\tau_{s}\right)-r\left(\tau_{s}\right)\right\}, s=1, \ldots, S,
$$

converge in distribution to independent identically distributed $N(0, G \psi(d))$ random variables, where

$$
\begin{aligned}
& \psi(0)=2 \pi \kappa(0), \\
& \psi(d)=\phi(d) \kappa(d), 0<|d|<\frac{1}{2},
\end{aligned}
$$

in which

$$
\begin{aligned}
\kappa(d) & =\int_{-\infty}^{\infty} \int_{-\infty}^{\infty} k(v)\{k(w)-k(v)\}|v-w|^{2 d-1} d v d w,-\frac{1}{2}<d<0 \\
\kappa(0) & =\int_{-\infty}^{\infty} k(v)^{2} d v \\
\kappa(d) & =\int_{-\infty}^{\infty} \int_{-\infty}^{\infty} k(v) k(w)|v-w|^{2 d-1} d v d w, 0<d<\frac{1}{2}
\end{aligned}
$$

The variance $G \psi(d)$ in the limit distribution is desirably constant over the $\tau_{s}$. This is in contrast to the outcome with kernel estimates of random-design nonparametric regression. Indeed, in that setting, with long memory the desirable independence in the limit distribution may also be lost, the limiting covariance matrix possibly even having only unit rank (see Robinson, 2007). Other asymptotic properties of kernel fixed-design nonparametric regression with long memory disturbances are discussed by Hall and Hart (1990), Csorgo and Mielniczuk (1995).

Application of the central limit theorem in statistical inference requires estimation of $G$ and $d$. Robinson (1997) justified local Whittle estimates of $G$ and $d$, based on residuals from the nonparametric regression, to the extent that replacing $G$ and $d$ by the estimates produces asymptotically valid inferences. 
This outcome relies also on the continuity of $\psi(d)$, demonstrated in Robinson (1997). The estimate of $d$ is simply substituted for $d$ in $\phi(d)$, while the version (1.16)-(1.18) of $\kappa($.$) used will automatically reflect the sign of the estimate of d$.

Calculation of $\kappa(d)$ is not discussed by Robinson (1997). For $d \neq 0$ a double integral is involved, which for general $k(u)$ cannot be obtained analytically. Moreover, some additional care is needed when $d<0$, in that $k(v) k(w)|v-w|^{2 d-1}$ and $k(v)^{2}|v-w|^{2 d-1}$ are not integrable individually though their difference is.

The present paper fills a gap by deducing analytic formulae for $\kappa(d)$ for a particular class of $k(u)$, namely (even) polynomials of arbitrary finite degree on $(-1,1)$. This class includes the uniform kernel, the "optimal" Epanechnikov kernel, and also covers bias-reducing higher-order kernels of arbitrary degree. (In an empirical example, Robinson (1997) employed an alternative specific cosine-bell - $k(u)$ which is not a member of this class and is not a higher-order kernel, and had to approximate $\kappa(d)$ numerically.) The results are presented in the following section, with proofs relegated to Section 4, these employing some lemmas established in Section 5. Section 3 illustrates the results, and discusses relevance to more general settings.

\section{Formulae for $\kappa(d)$}

We focus on $k(u)$ of form

$$
k(u)=\sum_{j=0}^{p} a_{j} u^{2 j},|u|<1 ;=0, \quad|u| \geq 1,
$$

for prescribed $p \geq 0$. When $p=0, k(u)$ is flat, and (1.3) implies $a_{0}=\frac{1}{2}$. When $p=1$, (1.3) implies $2 a_{0}+2 a_{1} / 3=1$, an important special case being the Epanechnikov mean-squared-error optimal kernel in which $a_{0}=\frac{3}{4}, a_{1}=-\frac{3}{4}$. When $p \geq 1$, higher-order kernels are a possibility, for example satisfying the constraints

$$
\int_{-\infty}^{\infty} u^{i} k(u) d u=0, \quad 1 \leq i \leq 2 p
$$

Thus, under (2.1), (2.2) we require

$$
\sum_{j=0}^{p} a_{j} \int_{-1}^{1} u^{2 j+i} d u=0, \quad 1 \leq i \leq 2 p
$$

that is

$$
\sum_{j=0}^{p} \frac{a_{j}}{2 j+2 \ell+1}=0, \quad 1 \leq \ell \leq p .
$$

Thus, imposing also (1.3), the vector $a=\left(a_{0}, \ldots, a_{p}\right)^{\prime}$ is given by

$$
a=B^{-1} b
$$


where the $(p+1) \times(p+1)$ matrix $B$ has $(i, j)$ th element

$$
B_{i j}=\frac{1}{2(i-1)+2(j-1)+1},
$$

and $b$ is the $(p+1) \times 1$ vector whose first element is 2 and whose remaining elements are 0 . For example if $p=1$, then $a_{0}=9 / 8, a_{1}=-15 / 8$. For $p>1$, we can generalize (2.2) by replacing $p$ there by $q \in[1, p]$. Of course, (2.1) excludes a number of popularly-used kernels, but except for the issue of kernel order the actual choice of kernel is often believed to be of secondary importance.

Theorem 1 For $d=0$, and $k(u)$ given by (2.1),

$$
\kappa(d)=2 \sum_{i=0}^{p} \sum_{j=0}^{p} \frac{a_{i} a_{j}}{2 i+2 j+1} .
$$

Define, for $b \in(-1,0)$ and even $r, s$,

$$
I(r, s ; b)=2 r ! s ! \Gamma(b+1) \sum_{\ell=0}^{r} \sum_{m=0}^{s} \frac{(-1)^{\ell+m} 2^{\ell+m+b+2}}{(r-\ell) !(s-m) ! \Gamma(\ell+m+b+3)} .
$$

Theorem 2 For $d \in(0,0.5)$, and $k(u)$ given by (2.1),

$$
\kappa(d)=\sum_{i=0}^{p} \sum_{j=0}^{p} a_{i} a_{j} I(2 i, 2 j ; 2 d-1) .
$$

Define, for $b \in(-2,-1)$, even $r$ and even $s \geq 2$,

$$
\begin{gathered}
J(r, s ; b)=\sum_{n=0}^{s-1}(-1)^{n} I(r+n, s-1-n ; b+1), \\
K(r ; b)=-2 r ! \Gamma(b+1) \sum_{\ell=0}^{r} \frac{(-1)^{\ell}}{(r-\ell) ! \Gamma(\ell+b+3)} 2^{\ell+b+2} .
\end{gathered}
$$

Theorem 3 For $d \in(-0.5,0)$, and $k(u)$ given by (2.1),

$$
\left.\kappa(d)=\sum_{i=0}^{p} \sum_{j=1}^{p} a_{i} a_{j} J(2 i, 2 j ; 2 d-1)\right) 1(p>0)-\sum_{i=0}^{p} \sum_{j=0}^{p} a_{i} a_{j} K(2 i+2 j ; 2 d-1) .
$$

\section{Discussion}

We illustrate the formulae first with $p=0$, whence $a_{0}=\frac{1}{2}$. From (2.3)

$$
\psi(0)=2 \pi \kappa(0)=2 \pi \times 2 a_{0}^{2}=\pi .
$$

From (2.4)

$$
I(0,0 ; 2 d-1)=\frac{2^{2 d+2}}{2 d(2 d+1)}
$$


Thus from (2.5)

$$
\begin{aligned}
\psi(d) & =2 \Gamma(1-2 d) \sin (\pi d) a_{0}^{2} I(0,0 ; 2 d-1) \\
& =\frac{1}{2} \Gamma(1-2 d) \sin (\pi d) \frac{2^{2 d+2}}{2 d(2 d+1)} \\
& =\Gamma(1-2 d) \sin (\pi d) \frac{2^{2 d}}{d(2 d+1)}, \quad 0<d<\frac{1}{2},
\end{aligned}
$$

and from $(2.8)$

$$
\begin{aligned}
\psi(d) & =-2 \Gamma(1-2 d) \sin (\pi d) a_{0}^{2} K(0 ; 2 d-1) \\
& =2 \Gamma(1-2 d) \sin (\pi d) a_{0}^{2} I(0,0 ; 2 d-1) \\
& =\Gamma(1-2 d) \sin (\pi d) \frac{2^{2 d}}{d(2 d+1)},-\frac{1}{2}<d<0 .
\end{aligned}
$$

Notice that as $d \rightarrow 0^{+}$, and $d \rightarrow 0^{-}, \psi(d) \rightarrow \psi(0)$.

Now take $p=1$. We have

$$
\kappa(0)=2 a_{0}^{2}+\frac{4 a_{0} a_{1}}{3}+\frac{2 a_{1}^{2}}{5},
$$

SO

$$
\psi(0)=2 \pi\left(2 a_{0}^{2}+\frac{4 a_{0} a_{1}}{3}+\frac{2 a_{1}^{2}}{5}\right) .
$$

Because the formulae become complicated we finally consider $\psi(d)$ for $0<d<$ $1 / 2$ only. Write

$$
\begin{aligned}
I(r, s ; b) & =2 r ! s ! \Gamma(b+1) M(r, s ; b) . \\
M(r, s ; b) & =\sum_{\ell=0}^{r} \sum_{m=0}^{s} \frac{(-1)^{\ell+m} 2^{b+2+\ell+m}}{(r-\ell) !(s-m) ! \Gamma(\ell+m+b+3)} .
\end{aligned}
$$

Then

$$
\begin{aligned}
M(2,0 ; b) & =\frac{2^{b+1}}{\Gamma(b+3)}-\frac{2^{b+3}}{\Gamma(b+4)}+\frac{2^{b+4}}{\Gamma(b+5)} \\
& =\frac{2^{b+1}}{\Gamma(b+5)}\{(b+3)(b+4)-4(b+4)+8\} \\
& =\frac{2^{b+1}\left(b^{2}+3 b+4\right)}{\Gamma(b+5)},
\end{aligned}
$$

so

$$
I(2,0 ; 2 d-1)=\frac{2^{2 d+2}\left(2 d^{2}+d+1\right)}{(2 d+3)(2 d+2)(2 d+1) d} .
$$

Next 


$$
\begin{aligned}
M(2,2 ; b)= & \frac{M(2,0 ; b)}{2}+\sum_{\ell=0}^{2} \frac{(-1)^{\ell+1} 2^{b+3+\ell}}{(2-\ell) ! \Gamma(\ell+b+4)}+\sum_{\ell=0}^{2} \frac{(-1)^{\ell+2} 2^{b+4+\ell}}{(2-\ell) ! \Gamma(\ell+b+5)} \\
= & \frac{2^{b}}{\Gamma(b+3)}-\frac{2^{b+2}}{\Gamma(b+4)}+\frac{2^{b+3}}{\Gamma(b+5)} \\
& -\frac{2^{b+2}}{\Gamma(b+4)}+\frac{2^{b+4}}{\Gamma(b+5)}-\frac{2^{b+5}}{\Gamma(b+6)} \\
& +\frac{2^{b+3}}{\Gamma(b+5)}-\frac{2^{b+5}}{\Gamma(b+6)}+\frac{2^{b+6}}{\Gamma(b+7)} \\
= & 2^{b}\left\{\frac{1}{\Gamma(b+3)}-\frac{2^{3}}{\Gamma(b+4)}+\frac{2^{5}}{\Gamma(b+5)}-\frac{2^{6}}{\Gamma(b+6)}+\frac{2^{6}}{\Gamma(b+7)}\right\} .
\end{aligned}
$$

Thus

$$
\begin{aligned}
I(2,2 ; 2 d-1)= & 8 \Gamma(2 d)\left\{\frac{2^{2 d-1}}{\Gamma(2 d+2)}-\frac{2^{2 d+2}}{\Gamma(2 d+3)}+\frac{2^{2 d+4}}{\Gamma(2 d+4)}\right. \\
& \left.-\frac{2^{2 d+5}}{\Gamma(2 d+5)}+\frac{2^{2 d+5}}{\Gamma(2 d+6)}\right\} .
\end{aligned}
$$

It follows that

$$
\begin{aligned}
\kappa(d)= & a_{0}^{2} I(0,0 ; 2 d-1)+2 a_{0} a_{1} I(2,0 ; 2 d-1)+a_{1}^{2} I(2,2 ; 2 d-1) \\
= & a_{0}^{2} \frac{2^{2 d+2}}{2 d(2 d+1)}+2 a_{0} a_{1} \frac{2^{2 d+2}\left(2 d^{2}+d+1\right)}{(2 d+3)(2 d+2)(2 d+1) d} \\
& +a_{1}^{2} 8 \Gamma(2 d)\left\{\frac{2^{2 d-1}}{\Gamma(2 d+2)}-\frac{2^{2 d+2}}{\Gamma(2 d+3)}+\frac{2^{2 d+4}}{\Gamma(2 d+4)}-\frac{2^{2 d+5}}{\Gamma(2 d+5)}+\frac{2^{2 d+5}}{\Gamma(2 d+6)}\right\} .
\end{aligned}
$$

Our results are relevant to more general models than (1.1). For example, we might consider an $N \times 1$ vector of observations $Y_{t}$ satisfying

$$
Y_{t}=R\left(\frac{t}{T}\right)+X_{t}, t=1, \ldots, T,
$$

where $R$ is an $N \times 1$ vector of smooth functions, and $X_{t}$ is a vector of zero-mean jointly covariance stationary processes. We can estimate each element of $R\left(\tau_{s}\right)$, $s=1, \ldots, S$, as in (1.2), and deduce from Robinson (1997) $N S$-dimensional central limit theorems. We can also consider a joint, $S N$-dimensional, central limit theorem for all the estimates, under suitable conditions on $X_{t}$. For each $\tau_{s}$, the asymptotic covariance matrix of the estimate of $R\left(\tau_{s}\right)$ will not be diagonal unless the elements of $X_{t}$ are incoherent. However, it will have scalar factor $\kappa(d)$, as above.

With panel data, where $N$ might be large, there is interest in a more parsimonious structure than (3.1). One example concerns a panel whose members share the same-shaped nonparametric regression function, but its location and magnitude can vary across individuals. Consider

$$
Y_{t}=\alpha+\beta r(t / T)+X_{t}, \quad t=1, \ldots, T,
$$

where $\alpha$ and $\beta$ are $N \times 1$ vectors, such that $\alpha \neq \beta$ and $\beta \neq 0$, and $X_{t}$ possibly exhibits spatial or other cross-sectional correlation. Because the location and 
scale of $r$ are unrestricted, we can, with no loss of generality, take $1^{\prime} \alpha=0$, $1^{\prime} \beta=1$, where 1 is the $N \times 1$ vector of ones. Thus the cross-sectional aggregates $1^{\prime} Y_{t}$ satisfy

$$
1^{\prime} Y_{t}=r(t / T)+1^{\prime} X_{t} .
$$

Thus, taking $y_{t}=1^{\prime} Y_{t}, x_{t}=1^{\prime} X_{t}$, we revert to the model (1.1). With respect to the cross-sectional properties of the elements of $X_{t}$, these may or may not have identical memory parameters. If they do, then $d$ is the common value, unless certain cointegration is present. If they do not, then generally the largest of them is $d$. When the memory parameters differ, this can influence the "local smoothness parameter" of $f(\lambda)$, but this is not of concern in our central limit theorem for the $\hat{r}\left(\tau_{s}\right)$, though it is relevant to asymptotic theory for estimates of $d$ (see Robinson, 1997).

\section{Proofs of Theorems}

Proof of Theorem 1 Straightforward.

Proof of Theorem 2 The result is clearly true by substitution if we can show that for even $r, s$ and for $b=2 d-1$,

$$
\int_{-1}^{1} \int_{-1}^{1} v^{r} w^{s}|v-w|^{b} d v d w=I(r, s ; b) .
$$

The left side is

$$
\begin{aligned}
& \int_{0}^{1} \int_{0}^{1} v^{r} w^{s}|v-w|^{b} d v d w+\int_{0}^{1} \int_{-1}^{0} v^{r} w^{s}|v-w|^{b} d v d w \\
& +\int_{-1}^{0} \int_{0}^{1} v^{r} w^{s}|v-w|^{b} d v d w+\int_{-1}^{0} \int_{-1}^{0} v^{r} w^{s}|v-w|^{b} d v d w \\
= & 2 \int_{0}^{1} \int_{0}^{1} v^{r} w^{s}|v-w|^{b} d v d w+2 \int_{0}^{1} \int_{-1}^{0} v^{r} w^{s}|v-w|^{b} d v d w \\
= & 2 \int_{0}^{1} w^{s}\{F(w ; r, b)+G(w ; r, b)+H(w ; r, b)\} d w,
\end{aligned}
$$

where

$F(w ; r, b)=\int_{0}^{w} v^{r}(w-v)^{b} d v, G(w ; r, b)=\int_{w}^{1} v^{r}(v-w)^{b} d v, H(w ; r, b)=\int_{0}^{1} v^{r}(w+v)^{b} d v$. 
From Lemmas 1-3, and Corollary 3, (4.1) is

$$
\begin{aligned}
& 2 \int_{0}^{1} w^{s}\left\{B(r+1, b+1) w^{r+b+1}+r ! \sum_{\ell=0}^{r} \frac{1}{(\ell+b+1)(r-\ell) ! \ell !} w^{r-\ell}(1-w)^{\ell+b+1}\right. \\
& \left.+r ! \Gamma(b+1) \sum_{\ell=0}^{r} \frac{(-1)^{\ell}}{(r-\ell) ! \Gamma(\ell+b+2)}(w+1)^{\ell+b+1}-B(r+1, b+1) w^{r+b+1}\right\} d w \\
= & 2 r !\left\{\sum_{\ell=0}^{r} \frac{1}{(\ell+b+1)(r-\ell) ! \ell !} \int_{0}^{1} w^{r+s-\ell}(1-w)^{\ell+b+1} d w\right. \\
& \left.+\Gamma(b+1) \sum_{\ell=0}^{r} \frac{(-1)^{\ell}}{(r-\ell) ! \Gamma(\ell+b+2)} H(1 ; s, \ell+b+1)\right\} \\
= & 2 r ! \sum_{\ell=0}^{r} \frac{B(r+s-\ell+1, \ell+b+2)}{(r-\ell) !(\ell+b+1) \ell !}+2 r ! \Gamma(b+1) \sum_{\ell=0}^{r} \frac{(-1)^{\ell}}{(r-\ell) ! \Gamma(\ell+b+2)} \\
& \times\left\{s ! \Gamma(\ell+b+2) \sum_{m=0}^{s} \frac{(-1)^{m}}{(s-m) ! \Gamma(\ell+m+b+3)} 2^{\ell+m+b+2}-B(s+1, \ell+b+2)\right\} . \\
= & \frac{2}{\Gamma(r+s+b+3)} \sum_{\ell=0}^{r} \frac{\Gamma(r+s-\ell+1) \Gamma(\ell+b+1)}{(r-\ell) ! \ell !}+2 r ! s ! \Gamma(b+1) \sum_{\ell=0}^{r} \frac{(-1)^{\ell}}{(r-\ell) !} \\
& \times\left\{\sum_{m=0}^{s} \frac{(-1)^{m}}{(s-m) ! \Gamma(\ell+m+b+3)} 2^{\ell+m+b+2}-\frac{1}{\Gamma(\ell+s+b+3)}\right\}
\end{aligned}
$$

for even $s$. Using a combinatorial identity (Knuth, 1968, p. 57, formula 18)

$$
\begin{aligned}
\sum_{\ell=0}^{r} \frac{(-1)^{\ell} \Gamma(r+s+b+3)}{(r-\ell) ! \Gamma(\ell+s+b+3)} & =\sum_{\ell=0}^{r} \frac{(-1)^{\ell} \Gamma(r+s+b+3)}{\ell ! \Gamma(r+s-\ell+b+3)} \\
& =(-1)^{r} \frac{\Gamma(r+s+b+2)}{r ! \Gamma(s+b+2)} \\
& =\frac{\Gamma(r+s+b+2)}{r ! \Gamma(s+b+2)}
\end{aligned}
$$

for even $r$. Using another combinatorial identity (Knuth, 1968, p. 58, formula 26 , with $t=-1$ ):

$$
\sum_{\ell=0}^{r} \frac{\Gamma(r+s-\ell+1) \Gamma(\ell+b+1)}{(r-\ell) ! \ell !}=\frac{s ! \Gamma(b+1) \Gamma(r+s+b+2)}{r ! \Gamma(s+b+2)} .
$$

Then (2.4) follows by substitution.

Proof of Theorem 3 Clearly $\kappa(d)$ is

$$
\begin{aligned}
& \int_{-\infty}^{\infty} \int_{-1}^{1} \sum_{i=0}^{p} a_{i} v^{2 i}\left\{\sum_{j=0}^{p} a_{j}\left(w^{2 j} 1(|w|<1)-v^{2 j}\right)\right\}|v-w|^{2 d-1} d v d w \\
= & \int_{-1}^{1} \int_{-1}^{1} \sum_{i=0}^{p} \sum_{j=1}^{p} a_{i} a_{j} v^{2 i}\left(w^{2 j}-v^{2 j}\right)|v-w|^{2 d-1} d v d w 1(p>0) \\
& -2 \int_{1}^{\infty} \int_{-1}^{1}\left(\sum_{j=0}^{p} a_{j} v^{2 j}\right)^{2}(w-v)^{2 d-1} d v d w,
\end{aligned}
$$


with $1($.$) denoting the indicator function. This can be written as (2.8)$ with, for $b<-1, r \geq 0, s \geq 1$,

$$
\begin{aligned}
J(r, s ; b) & =\int_{-1}^{1} \int_{-1}^{1} v^{r}\left(w^{s}-v^{s}\right)|v-w|^{b} d v d w, \\
K(r ; b) & =2 \int_{1}^{\infty} \int_{-1}^{1} v^{r}(w-v)^{b} d v d w,
\end{aligned}
$$

whence it remains to show that definitions (2.6) and (2.7) hold.

For even $r, s$, with $s \geq 2$,

$$
\begin{aligned}
J(r, s ; b)= & \int_{0}^{1} \int_{0}^{1} v^{r}\left(w^{s}-v^{s}\right)|v-w|^{b} d v d w+\int_{0}^{1} \int_{-1}^{0} v^{r}\left(w^{s}-v^{s}\right)|v-w|^{b} d v d w \\
& +\int_{-1}^{0} \int_{0}^{1} v^{r}\left(w^{s}-v^{s}\right)|v-w|^{b} d v d w+\int_{-1}^{0} \int_{-1}^{0} v^{r}\left(w^{s}-v^{s}\right)|v-w|^{b} d v d w \\
= & 2 \int_{0}^{1} \int_{0}^{1} v^{r}\left(w^{s}-v^{s}\right)|v-w|^{b} d v d w+2 \int_{0}^{1} \int_{-1}^{0} v^{r}\left(w^{s}-v^{s}\right)(w-v)^{b} d v d w .
\end{aligned}
$$

Now

$$
\begin{aligned}
\int_{0}^{1} \int_{0}^{1} v^{r}\left(w^{s}-v^{s}\right)|v-w|^{b} d v d w & =\int_{0}^{1} \int_{0}^{1} v^{r} \sum_{n=0}^{s-1} v^{n} w^{s-n-1}(w-v)|v-w|^{b} d v d w \\
& =\sum_{n=0}^{s-1} \int_{0}^{1} w^{s-n-1}\{F(w ; r+n, b+1)-G(w ; r+n, b+1)\} d w .
\end{aligned}
$$

From Lemma 1,

$$
F(w ; r+n, b+1)=w^{r+n+b+2} B(r+n+1, b+2) .
$$

From Lemma 2,

$$
G(w ; r+n, b+1)=(r+n) ! \sum_{\ell=0}^{r+n} \frac{1}{(\ell+b+2)(r+n-\ell) ! \ell !} w^{r+n-\ell}(1-w)^{\ell+b+2} .
$$

Thus

$$
\int_{0}^{1} w^{s-1-n}\{F(w ; r+n, b+1)-G(w ; r+n, b+1)\} d w
$$

is

$$
\begin{aligned}
& B(r+n+1, b+2) \int_{0}^{1} w^{r+s+b+1} d w \\
& -(r+n) ! \sum_{\ell=0}^{r+n} \frac{1}{(\ell+b+2)(r+n-\ell) ! \ell !} \int_{0}^{1} w^{r+s-\ell-1}(1-w)^{b+\ell+2} d w \\
= & \frac{B(r+n+1, b+2)}{r+s+b+2}-(r+n) ! \sum_{\ell=0}^{r+n} \frac{B(r+s-\ell, \ell+b+3)}{(\ell+b+2)(r+n-\ell) ! \ell !} \\
= & \frac{B(r+n+1, b+2)}{r+s+b+2}-\frac{(r+n) !}{\Gamma(r+s+b+3)} \sum_{\ell=0}^{r+n} \frac{\Gamma(r+s-\ell) \Gamma(\ell+b+2)}{(r+n-\ell) ! \ell !} .
\end{aligned}
$$


Using again (Knuth, 1968, p. 58, formula 26, with $t=-1$ )

$$
\sum_{\ell=0}^{r+n} \frac{\Gamma(r+s-\ell) \Gamma(\ell+b+2)}{(r+n-\ell) ! \ell !}=\frac{\Gamma(b+2) \Gamma(r+s+b+2)(s-n-1) !}{(r+n) ! \Gamma(s-n+b+2)},
$$

so $(4.2)$ is

$$
\begin{aligned}
& \frac{B(r+n+1, b+2)}{r+s+b+2}-\frac{\Gamma(b+2)(s-n-1) !}{(r+s+b+2) \Gamma(s-n+b+2)} \\
= & \frac{B(r+n+1, b+2)-B(s-n, b+2)}{r+s+b+2} .
\end{aligned}
$$

Thus

$$
\begin{aligned}
& \sum_{n=0}^{s-1} \int_{0}^{1} w^{s-1-n}\{F(w ; r+n, b+1)-G(w ; r+n, b+1\} d w \\
= & \frac{1}{r+s+b+2} \sum_{n=0}^{s-1}\{B(r+n+1, b+2)-B(s-n, b+2)\} .
\end{aligned}
$$

Next

$$
\begin{aligned}
\int_{0}^{1} \int_{-1}^{0} v^{r}\left(w^{s}-v^{s}\right)(w-v)^{b} d v d w & =\int_{0}^{1} \int_{0}^{1} v^{r}\left(w^{s}-v^{s}\right)(w+v)^{b} d v d w \\
\cdot & =\int_{0}^{1} \int_{0}^{1} v^{r} \sum_{n=0}^{s-1}(-v)^{n} w^{s-n-1}(w+v)^{b+1} d v d w \\
& =\sum_{n=0}^{s-1}(-1)^{n} \int_{0}^{1} \int_{0}^{1} v^{r+n} w^{s-n-1}(w+v)^{b+1} d v d w \\
& =\sum_{n=0}^{s-1}(-1)^{n} \int_{0}^{1} w^{s-n-1} H(w ; r+n, b+1) d w
\end{aligned}
$$

From Lemma 3,

$$
\begin{aligned}
& H(w ; r+n, b+1)=(r+n) ! \Gamma(b+2) \sum_{\ell=0}^{r+n} \frac{(-1)^{\ell}}{(r+n-\ell) ! \Gamma(\ell+b+3)}(w+1)^{\ell+b+2} \\
& -(-1)^{r+n} B(r+n+1, b+2) w^{r+n+b+2},
\end{aligned}
$$

so since $r$ is even the integral is

$$
\begin{aligned}
& (r+n) ! \Gamma(b+2) \sum_{\ell=0}^{r+n} \frac{(-1)^{\ell}}{(r+n-\ell) ! \Gamma(\ell+b+3)} H(1 ; s-n-1, \ell+b+2) \\
& -(-1)^{n} B(r+n+1, b+2) \int_{0}^{1} w^{r+s+b+1} d w .
\end{aligned}
$$

From Corollary 3,

$$
\begin{aligned}
H(1 ; s-n-1, \ell+b+2)= & (s-n-1) ! \Gamma(b+3+\ell) \sum_{m=0}^{s-n-1} \frac{(-1)^{m}}{(s-n-1) ! \Gamma(\ell+m+b+4)} 2^{\ell+m+b+3} \\
& -(-1)^{s-n-1} B(s-n, b+3+\ell) .
\end{aligned}
$$


Thus

$$
\begin{aligned}
& \sum_{\ell=0}^{r+n} \frac{(-1)^{\ell}}{(r+n-\ell) ! \Gamma(\ell+b+3)} H(1 ; s-n-1, \ell+b+2) \\
= & (s-n-1) ! \sum_{\ell=0}^{r+n} \frac{(-1)^{\ell}}{(r+n-\ell) !} \sum_{m=0}^{s-n-1} \frac{(-1)^{m} 2^{\ell+m+b+3}}{(s-n-m-1) ! \Gamma(\ell+m+b+4)} \\
& -\sum_{\ell=0}^{r+n} \frac{(-1)^{\ell+s-n-1}}{(r+n-\ell) ! \Gamma(\ell+b+3)} B(s-n, \ell+b+3),
\end{aligned}
$$

and so

$$
\begin{aligned}
& (r+n) ! \Gamma(b+2) \sum_{\ell=0}^{r+n} \frac{(-1)^{\ell}}{(r+n-\ell) ! \Gamma(\ell+b+3)} H(1 ; s-n-1, \ell+b+2) \\
= & \Gamma(b+2)(r+n) !(s-n-1) ! \sum_{\ell=0}^{r+n} \sum_{m=0}^{s-n-1} \frac{(-1)^{\ell+m} 2^{\ell+m+b+3}}{(r+n-\ell) !(s-n-m-1) ! \Gamma(\ell+m+b+4)} \\
& -\Gamma(b+2)(r+n) ! \sum_{\ell=0}^{r+n} \frac{(-1)^{\ell+s-n-1}}{(r+n-\ell) ! \Gamma(\ell+b+3)} B(s-n, \ell+b+3) \\
= & \frac{1}{2} I(r+n, s-n-1, b+1)-\Gamma(b+2)(r+n) ! \sum_{\ell=0}^{r+n} \frac{(-1)^{\ell+s-n-1} \Gamma(s-n)}{(r+n-\ell) ! \Gamma(\ell+s-n+b+3)} .
\end{aligned}
$$

From Knuth (1968, p.57, formula 18) again,

$$
\begin{aligned}
\sum_{\ell=0}^{r+n} \frac{(-1)^{\ell} \Gamma(r+s+b+3)}{(r+n-\ell) ! \Gamma(\ell+s-n+b+3)} & =\sum_{\ell=0}^{r+n} \frac{(-1)^{r+n-\ell} \Gamma(r+s+b+3)}{\ell ! \Gamma(r+s-\ell+b+3)} \\
& =\frac{(-1)^{r+n} \Gamma(r+s+b+2)}{(r+n) ! \Gamma(s-n+b+2)},
\end{aligned}
$$

so

$$
\begin{aligned}
& \Gamma(b+2)(r+n) ! \Gamma(s-n) \sum_{\ell=0}^{r+n} \frac{(-1)^{\ell+s-n-1}}{(r+n-\ell) ! \Gamma(\ell+s-n+b+3)} \\
= & \Gamma(b+2) \frac{(r+n) ! \Gamma(s-n)(-1)^{s-n-1}}{(r+n) ! \Gamma(s-n+b+2)} \frac{\Gamma(r+s+b+2)}{\Gamma(r+s+b+3)} \\
= & -\frac{(-1)^{n} \Gamma(b+2) \Gamma(s-n)}{\Gamma(s-n+b+2)(r+s+b+2)}=-(-1)^{n} \frac{B(s-n, b+2)}{r+s+b+2},
\end{aligned}
$$

since $s$ is even. Also

$$
B(r+n+1, b+2) \int_{0}^{1} w^{r+s+b+1} d v=\frac{B(r+n+1, b+2)}{r+s+b+2} .
$$

It follows that

$$
\begin{aligned}
\int_{0}^{1} w^{s-n-1} H(w ; r+n, b+1) d w= & \frac{1}{2} I(r+n, s-n-1 ; b+1) \\
& +(-1)^{n} \frac{B(s-n, b+2)-B(r+n+1, b+2)}{r+s+b+2} .
\end{aligned}
$$


Then from (4.3)

$$
\begin{aligned}
J(r, s ; b)= & \frac{2}{r+s+b+2} \sum_{n=0}^{s-1}\{B(r+n+1, b+2)-B(s-n, b+2)\} \\
& +\sum_{n=0}^{s-1}(-1)^{n} I(r+n, s-n-1 ; b+1) \\
& +\frac{2}{r+s+b+2} \sum_{n=0}^{s-1}(-1)^{2 n}\{B(s-n, b+2)-B(r+n+1, b+2)\} \\
= & \sum_{n=0}^{s-1}(-1)^{n} I(r+n, s-n-1 ; b+1),
\end{aligned}
$$

giving (2.6). Next, for even $r$ and $b<-1$,

$$
\begin{aligned}
K(r ; b)= & 2 \int_{-1}^{1} v^{r}\left\{\int_{1}^{\infty}(w-v)^{b} d w\right\} d v \\
= & 2 \int_{-1}^{1} v^{r}\left[\frac{(w-v)^{b+1}}{b+1}\right]_{1}^{\infty} d v \\
= & -\frac{2}{b+1} \int_{-1}^{1} v^{r}(1-v)^{b+1} d v \\
= & -\frac{2}{b+1} \int_{0}^{1} v^{r}(1-v)^{b+1} d v-\frac{2}{b+1} H(1 ; r, b+1) \\
= & -\frac{2}{b+1} B(r+1, b+2) \\
& -\frac{2}{b+1}\left\{r ! \Gamma(b+2) \sum_{m=0}^{r} \frac{(-1)^{m}}{(r-m) ! \Gamma(m+b+3)} 2^{m+b+2}-B(r+1, b+2)\right\} \\
= & -2 r ! \Gamma(b+1) \sum_{m=0}^{r} \frac{(-1)^{m}}{(r-m) ! \Gamma(m+b+3)} 2^{m+b+2},
\end{aligned}
$$

using Corollary 3, to prove (2.7).

\section{Technical Lemmas}

We state and prove three lemmas.

Lemma 1 For real $b>-1, r>-1$ and $w \in(0,1)$,

$$
F(w ; r, b)=w^{r+b+1} B(r+1, b+1) .
$$

Proof Follows from the definition of the Beta function after change of variable.

Lemma 2 For real $b \geq 0$, integer $r$ and $w \in(0,1)$,

$$
G(w ; r, b)=r ! \sum_{\ell=0}^{r} \frac{1}{(\ell+b+1)(r-\ell) !} w^{r-\ell}(1-w)^{\ell+b+1} .
$$


Proof We have

$G(w ; r, b)=\int_{0}^{1-w}(u+w)^{r} u^{b} d u=w^{r}(1-w)^{b+1} B(1, b+1)_{2} F_{1}(-r, b+1 ; b+2 ;(w-1) / w)$,

from Gradshteyn and Ryzhik (1994, p.335, formula 8), the last factor being the hypergeometric series

$$
\begin{aligned}
\left.{ }_{2} F_{1}(-r, b+1 ; b+2 ;(w-1) / w)\right)= & \frac{\Gamma(b+2)}{\Gamma(-r) \Gamma(b+1)} \sum_{j=0}^{\infty} \frac{\Gamma(j-r) \Gamma(j+b+1)}{\Gamma(j+b+2)} \frac{\{(w-1) / w\}^{j}}{j !} \\
= & 1-\frac{r(b+1)}{b+2}\left(\frac{w-1}{w}\right)-\frac{r(1-r)(b+1)(b+2)}{2(b+2)(b+3)}\left(\frac{w-1}{w}\right)^{2} \\
& -\frac{r(1-r)(2-r)(b+1)(b+2)(b+3)}{2 \times 3(b+2)(b+3)(b+4)}\left(\frac{w-1}{w}\right)^{3}-\ldots \\
& -\frac{r(1-r)(2-r) \ldots(r-1-r)(b+1)}{2 \times 3 \times \ldots \times r(r+b+1)}\left(\frac{w-1}{w}\right)^{r} \\
= & (b+1) r ! \sum_{\ell=0}^{r} \frac{1}{(\ell+b+1) \ell !(r-\ell) !}\left(\frac{1-w}{w}\right)^{\ell},
\end{aligned}
$$

see Gradshteyn and Ryzhik (1994, p.1065), whence the result follows from $B(1, b+1)=(b+1)^{-1}$.

Lemma 3 For real $b>-1$, integer $r$ and $w \in(0,1)$,

$H(w ; r, b)=r ! \Gamma(b+1) \sum_{\ell=0}^{r} \frac{(-1)^{\ell}}{(r-\ell) ! \Gamma(\ell+b+2)}(w+1)^{\ell+b+1}-(-1)^{r} B(r+1, b+1) w^{r+b+1}$.

Proof Successively integrating by parts,

$$
\begin{aligned}
H(w ; r, b)= & {\left[v^{r} \frac{(w+v)^{b+1}}{b+1}\right]_{0}^{1}-\frac{r}{b+1} \int_{0}^{1} v^{r-1}(w+v)^{b+1} d v } \\
= & \frac{(w+1)^{b+1}-w^{b+1} 1(r=0)}{b+1}-\frac{r}{b+1}\left[v^{r-1} \frac{(w+v)^{b+2}}{b+2}\right]_{0}^{1} \\
& +\frac{r(r-1)}{(b+1)(b+2)} \int_{0}^{1} v^{r-2}(w+v)^{b+2} d v \\
= & \frac{(w+1)^{b+1}-w^{b+1} 1(r=0)}{b+1}-\frac{r\left\{(w+1)^{b+2}-w^{b+2} 1(r=1)\right\}}{(b+1)(b+2)} \\
& +\frac{r(r-1)}{(b+1)(b+2)}\left[v^{r-2} \frac{(w+v)^{b+3}}{b+3}\right]_{0}^{1}-\frac{(b+1)(b+2)(b+3)}{(b+1)(r-2)} \int_{0}^{1} v^{r-3}(w+v)^{b+3} d v \\
= & \frac{(w+1)^{b+1}-w^{b+1} 1(r=0)}{b+1}-\frac{r\left\{(w+1)^{b+2}-w^{b+2} 1(r=1)\right\}}{(b+1)(b+2)} \\
& +\frac{r(r-1)\left\{(w+1)^{b+3}-w^{b+3} 1(r=2)\right\}}{(b+1)(b+2)(b+3)}-\ldots,
\end{aligned}
$$


giving the result.

The following corollary is also used.

Corollary 3 For real $b>-1$ and integer $r$,

$$
H(1 ; r, b)=r ! \Gamma(b+1) \sum_{\ell=0}^{r} \frac{(-1)^{\ell}}{(r-\ell) ! \Gamma(\ell+b+2)} 2^{\ell+b+1}-(-1)^{r} B(r+1, b+1) .
$$

\section{References}

Csorgo, S. \& J. Mielniczuk (1995). Nonparametric regression under longrange dependent normal errors. Annals of Statistics 23, 1000-1014.

Fox, R. \& M.S. Taqqu (1985). Non-central limit theorems for quadratic forms in random variables having long-range dependence. Annals of Probability $13,428-446$.

Gradshteyn, I.R. \& I.M. Ryzhik (1994). Table of Integrals, Series and Products. Academic Press.

Hall P. \& J.D. Hart (1990). Nonparametric regression with long-range dependence. Stochastic Processes and Their Applications 36, 339-351.

Knuth, D. E. (1968). The Art of Computer Programming. 1. Fundamental Algorithms. Addison-Wesley.

Robinson, P.M. (1997). Large-sample inference for nonparametric regression with dependent errors. Annals of Statistics 28, 2054-2083.

Robinson, P.M. (2007). Nonparametric regression with spatial data. Submitted for publication.

Roussas, G., L.T. Tran \& O. A. Ioannides (1992). Fixed design regression for time series: asymptotic normality. Journal of Multivariate Analysis 40, 262-291.

Yong, C. H. (1974). Asymptotic Behaviour of Trigonometric Series. Chinese University of Hong Kong. 